\title{
Exercise left ventricular outflow tract obstruction
} as a cause of exercise intolerance: combined stress echocardiography and cardiopulmonary
exercise testing

\author{
Krzysztof Smarż, Beata Zaborska, Tomasz Jaxa-Chamiec, Andrzej Budaj \\ Department of Cardiology, Postgraduate Medical School, Grochowski Hospital, Warsaw, Poland
}

In symptomatic patients with hypertrophic cardiomyopathy (HCM) without significant left ventricular outflow tract (LVOT) obstruction at rest, both cardiopulmonary exercise test (CPET) and exercise stress echocardiography are recommended [1] but they are usually performed separately. We propose the application of combined stress echocardiography and CPET in such cases. A 50-year-old man was admitted to our department because of two-week worsening of exercise capacity to New York Heart Association class II/III, exercise-induced chest pain, and heart palpitation on exertion. His history included the following: HCM without diagnosed LVOT obstruction, paroxysmal atrial fibrillation, arterial hypertension, chronic kidney disease, hyperlipidaemia, obesity, and smoking. At presentation the patient was in good clinical condition with resting heart rate (HR) of $60 \mathrm{bpm}$, blood pressure (BP) $140 / 80 \mathrm{mmHg}$, and body mass index $31 \mathrm{~kg} / \mathrm{m}^{2}$. Baseline laboratory tests indicated normal blood count and electrolyte levels, elevated creatinine level $(2.06 \mathrm{mg} / \mathrm{dL}$, estimated glomerular filtration rate $37 \mathrm{~mL} / \mathrm{kg} / 1.73 \mathrm{~m}^{2}$ ), and N-terminal pro-B-type natriuretic peptide level of $559.5 \mathrm{ng} / \mathrm{mL}$. The patient was treated with furosemide, bisoprolol, ramipril, spironolactone, atorvastatin, fenofibrate, and acenocumarol. In electrocardiogram, sinus rhythm and left ventricular hypertrophy (LVH) were present. Resting echocardiography showed significant LVH (interventricular septum: $24 \mathrm{~mm}$, posterior wall: $17 \mathrm{~mm}$ ) without LVOT obstruction, normal left vetricular contraction (ejection fraction 60\%), impaired relaxation (grade I), good right ventricular systolic function, slight left atrial enlargement, and mild mitral and tricuspid regurgitations. Chest X-ray revealed moderate cardiac enlargement without lung fluid. To assess the exercise capacity and cardiac function on exertion, exercise stress echocardiography and CPET were simultaneously performed. We used a semi-supine cycle ergometer and a protocol with loading fitted to the patient's functional status, to maintain exercise duration between 6 and $12 \mathrm{~min}$. The ramp protocol with loading of $25 \mathrm{~W}$ per 2 min was applied. Maximal predicted load for this patient was $233 \mathrm{~W}$ and maximal predicted HR was $170 \mathrm{bpm}$. Exercise was terminated after $7 \mathrm{~min}$ and $20 \mathrm{~s}$ at a workload of $74 \mathrm{~W}$ and HR of $77 \mathrm{bpm}(27 \%$ and $45 \%$ of the maximal predicted values, respectively) because of excessive fatigue (eight points in the 10-point Borg scale), without arrhythmia and electrocardiographic signs of ischaemia. The patient reported slight chest discomfort at peak exercise. Respiratory exchange ratio at peak exercise was 1.07 , peak oxygen uptake was $12.6 \mathrm{~mL} / \mathrm{kg} / \mathrm{min}$, and minute ventilation to carbon dioxide production slope was 33. Systolic BP increased from $130 \mathrm{mmHg}$ at rest to $150 \mathrm{mmHg}$ at peak exercise. Patient's functional status was classified as Weber class C and ventilatory class II. In echocardiography before exercise, LVOT obstruction without significant gradient $(46 \mathrm{mmHg})$ was noticed. At peak exercise LVOT gradient was significantly elevated $(120 \mathrm{mmHg}$ ) and systolic anterior motion of the anterior mitral valve leaflet was observed (Fig. 1). During exercise hyperkinetic left ventricular contraction was present without regional wall motion abnormalities (Suppl. Video 1 and 2 - see journal website). Combined stress echocardiography and CPET in the presented patient revealed that exercise intolerance was caused by exercise-induced LVOT obstruction with a low rise in the systolic BP and also with coexisting chronotropic incompetence. Stress echocardiography performed simultaneously with CPET has a promising utility in regard to the comprehensive assessment of patients with HCM. Stress echocardiography can unmask significant LVOT
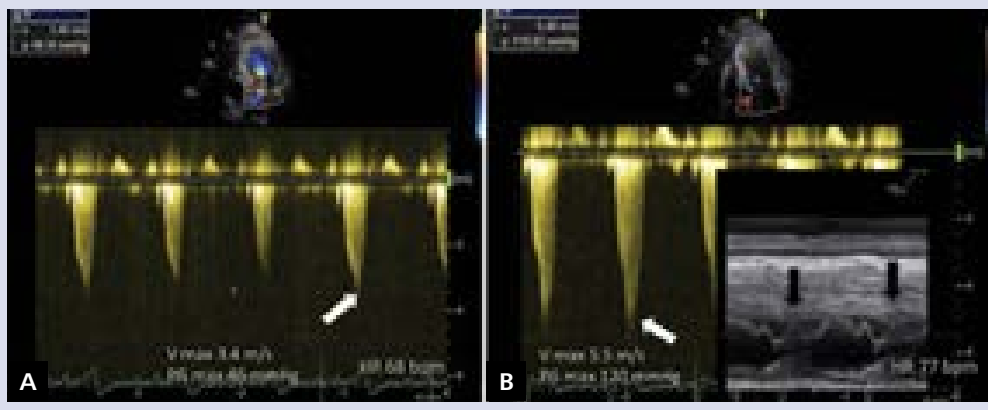

Figure 1. Left ventricular outflow tract maximal velocity ( $V$ max) and peak gradient (PG max) (continuous-wave Doppler) at rest (A) and at peak exercise (B) - white arrows. Systolic anterior motion was seen only at peak exercise (M-mode) - black arrows (B); HR - heart rate gradient $(\geq 50 \mathrm{mmHg}$ ), ventricular dysfunction, and valvular insufficiency. CPET allows the measurement of exercise capacity, pulmonary function, electrocardiographic changes, and BP response to exercise. Combined test enables to obtain a wide range of diagnostic and pathophysiological interactions of potentially important clinical value.

\section{References}

Elliott PM, Anastasakis A, Borger MA, et al. 2014 ESC Guidelines on diagnosis and management of hypertrophic cardiomyopathy: the Task Force for the Diagnosis and Management of Hypertrophic Cardiomyopathy of the European Society of Cardiology (ESC). Eur Heart J 2014; 35(39): 2733-2779, doi: 10.1093/eurhearti/ehu284, indexed in Pubmed: 25173338.

\section{Address for correspondence:}

Krzysztof Smarż, MD, Department of Cardiology, Postgraduate Medical School, Grochowski Hospital, ul. Grenadierów 51/59, 04-073 Warszawa, Poland, tel/fax: +48 2281017 38, e-mail: krzysztofsmarz@hotmail.com

Conflict of interest: none declared

Kardiologia Polska Copyright (c) Polish Cardiac Society 2018 